\title{
CHARACTERIZING DRIVER TAKE-OVER ACCURACY: EFFECT OF AGE, SEX, STARTLE, AND SECONDARY TASK
}

\author{
Valentina Graci $^{1}$, Meta Austin ${ }^{1,2}$, Madeline Griffith ${ }^{1}$, Rahul Akkem ${ }^{1}$, Jalaj Maheshwari ${ }^{1}$, \\ Thomas Seacrist $^{1}$, and Kristy B. Arbogast ${ }^{1}$ \\ ${ }^{1}$ Center for Injury Research and Prevention, Children's Hospital of Philadelphia, Philadelphia, PA,USA. \\ ${ }^{2}$ Brown University School of Engineering, Providence, RI,USA.
}

Corresponding Author: Valentina Graci, $\mathrm{PhD}$

2716 South St Floor 13 Rm\#13323

Tel: +1 267-425-0333

Email: graciv@chop.edu

https://doi.org/10.34107/BiomedSciInstrum.57.04281

\begin{abstract}
The Acoustic Startling Pre-stimulus (ASPS, i.e. a loud sound preceding a physical perturbation was previously found toacceleratetake-over actions in adults but not teens in autonomous vehicle scenarios. It is not clear if the ASPS also influences the accuracy of the take-over response across ages and sexes. Therefore the aims of this study are: to characterize take-over accuracy across age/experience andsexand to examine the effect of the ASPS and a secondary task on steering wheel alignment in autonomous vehicle take-over scenarios.

Fourteen adult ( 7 males and 13 teenage ( 6 males drivers volunteered for this study. Participants were instructed to align a marker on the steering wheel with a marker on a lateral post as fast as they could, when a sled perturbation started. Two of the conditions included the ASPS. Two of the conditions involved mobile texting while the sled started moving. The angle between the steering wheel and the lateral post was used to quantify overshooting, undershooting, or correct alignment during steering. Results showed thatadultfemale subjects reached correct alignment slightly more frequently than any other group, while male adult drivers decreased their alignment error after the first trial. Both female and male adult drivers had a reduced alignment angle when the first trial had an ASPS compared to when the first trial had no ASPS while teen drivers performed similarly with ASPS or without. This study showed that take-over accuracy and steering control are influenced by sex, age/experience,anda startle-based warning.
\end{abstract}

Keywords: autonomous vehicle, crash-avoidance maneuvers,steering,overshooting, undershooting.

\section{INTRODUCTION}

As autonomous vehicles become a reality, there will be instances in which drivers need to take over control of the vehicle to perform a crash-avoidance maneuver. Distractions will also be common since automation will allow engagement in secondary tasks [1], so that decreasing reaction time when taking over the control of the vehicle is warranted. Take-over time issues may be particularly relevant for inexperienced drivers, such as the recently licensed teen population, as $60 \%$ of teen driving errors have also been found to be due to inadequate surveillance and distractions [2]. In this population, the interaction of age (e.g. an immature brain) and limited experience makes them particularly at risk [3]. In order to decrease take-over reaction time (i.e. the difference between the onset of the warning and the instant the hands are on the steering wheel) several types of warnings have been examined by previous authors in studies where the driver was ready to act. Warnings were visual, auditory, or haptic. The resulting take-over reaction time showed great variability among studies and ranged between 1.14 to 8.7 seconds [1]. It was found that when a visual warning was paired with an acoustic warning, take-over 